\title{
Reactionless Visual Servoing of a Dual-Arm Space Robot
}

\author{
A. H. Abdul Hafez, V. V. Anurag, S. V. Shah, K. Madhava Krishna, and C. V. Jawahar
}

\begin{abstract}
This paper presents a novel visual servoing controller for a satellite mounted dual-arm space robot. The controller is designed to complete the task of servoing the robot's endeffectors to the desired pose, while regulating orientation of the base-satellite. Task redundancy approach is utilized to coordinate the servoing process and attitude of the base satellite. The visual task is defined as a primary task, while regulating attitude of the base satellite to zero is defined as a secondary task. The secondary task is formulated as an optimization problem in such a way that it does not affect the primary task, and simultaneously minimizes its cost function. A set of numerical experiments are carried out on a dual-arm space robot showing efficacy of the proposed control methodology.
\end{abstract}

\section{INTRODUCTION}

One of the major areas in space science that demands for immediate attention and commercial drive is the On Orbit Services (OSS) [1], e.g., orbital detritus management, refurbishment and refuelling of orbiting satellites, construction in space, etc. The OSS can be divided into rendezvous, proximity operations, and servicing. Use of robots boosts reliability, safety and ease of execution of operations during or after proximity operations [2]. It is highly desired that the closing over manoeuvre of the robot is carried out autonomously due to communication time delay between the service satellite and ground station [3]. This calls for a control technique which makes use of the on-board machine vision facility for successfully completing OOS in an autonomous manner. Visual servoing is one such visionbased technique commonly used for control of the earthbased robots, and have made many recent progresses [4], [5], [6], [7]. In this work, we attempt to solve a visual servoing problem for a satellite mounted space robot.

Visual servoing is inevitable for the space robots due to communication time delay [3]. Moreover, variable light conditions, absence of marker, and limited computing power make visual servoing further challenging [8]. Visual servoing was demonstrated for autonomous satellite capture in [3]. Later, they [8] showed visual servoing under limited availability of computing power and severe lighting conditions. Earth-based experimental evaluation for robotic capture of a helium airship using visual servoing was illustrated in [9]. There, the objective was to emulate capture of a free-floating object without emphasizing on effect of the robot's motion

\footnotetext{
* This research was supported by INSPIRE research Grant (IFA-13 ENG52) by Department of Science and Technology, India.

A. H. Abdul Hafez was with International Institute of Information Technology, Hyderabad - 500032, India, during this work. He is now with Hasan Kalyoncu University, Sahinbey - 27410, Gaziantep, Turkey

V. V. Anurag, S. V. Shah, K. Madhava Krishna, and C. V. Jawahar are with International Institute of Information Technology, Hyderabad - 500032, India
}

on the satellite. In the above works [3], [8], [9], satellite is either assumed to be controlled or operated in the freefloating mode. Use of attitude controller will consume fuel which is reserved mainly for orbital manoeuvres.

Robot's operation in the free-floating mode using the Generalized Jacobian Matrix (GJM) [10] is an another alternative. This although helps in reducing fuel consumption but causes change in the orientation of the base satellite. This may destabilise the satellite, cause damage to its internal hardware, and result in loss of communication with data relay satellite or ground station. Regulating attitude of the base satellite to zero in the free-floating mode is another objective of this work along with visual servoing.

In this regard, several researchers have focused on robotic manipulation with minimum attitude disturbance of the base satellite. This is also known as reactionless manipulation of robotic arm. This not only helps in keeping attitude disturbances minimum but also results into fuel saving. In this regard, the disturbance maps were proposed [11] to minimise change in the base attitude, but were not able to completely eliminate it. A Reaction Null Space (RNS) based formulation was also proposed in [12] which led to zero attitude disturbances of the base satellite. Recently, strategies for reactionless capture of tumbling objects using a dualarm robot were proposed in [13]. These works, however, stressed on reactionless path planning without emphasizing on vision-based control. In this work, we introduce a visionbased control for reactionless manipulation of a dual-arm robot. In our proposal, both visual servoing and reactionless manipulation are treated as different tasks. Multi-task approach was presented in [14] for visual servoing of an earth-based Cartesian robot with joint-limit and singularity avoidance as the additional tasks using gradient projection method. Success of such approach depends on proper tuning of a parameter that decides amplitude of secondary task. In order to overcome this disadvantage, an iterative scheme was presented for joint limit avoidance as a secondary task in [15]. Both the above methods required free degrees-offreedom (DOF) for secondary task. An improved task function approach was illustrated in [16], where the secondary task exploits DOF constrained by the main task in addition to the redundant DOF for faster completion of the task. The success of task functional approach on the earth-based robots inspired to use it for space robot.

However, implementation of visual servoing as the primary task and reactionless manipulation as the secondary task is a non-trivial problem for space robot mainly due to, 1) dynamic coupling between two arms and satellite, and 2) nonholonomic nature of the constraints for reactionless 
manipulation. In this work, a solution is proposed for the first two problems, which form the fundamental contributions of this work. The efficacy of the proposed approach is illustrated using a 6-DOF planar dual-arm robotic system mounted on a service satellite.

The rest of the paper is organized as follows: The Generalized Jacobian for a dual-arm space robot is presented in Section II. Visual servoing using the GJM is presented in Section III, whereas reactionless visual servoing is discussed in Section IV, Section $\mathrm{V}$ presents results and discussion. Finally, conclusions are given in Section VI.

\section{The Generalized Jacobian Matrix (GJM)}

In the proposed visual servoing, the base satellite is assumed to be free floating, and hence, it is very important to obtain the Jacobian that maps joint velocities into the end-effectors' velocities by incorporating motion of the base satellite. For an $n$-DOF dual-arm robotic system mounted on a floating-base with 2-end-effectors, as shown in Fig. 1, the end-effectors' velocities $\left(\boldsymbol{t}_{e}=\left[\begin{array}{ll}\boldsymbol{t}_{e 1}{ }^{T} & \boldsymbol{t}_{e 2}{ }^{T}\end{array}\right]^{T} \in R^{12}\right)$ are expressed in terms of the base velocity and joint velocity as

$$
\boldsymbol{t}_{e}=\boldsymbol{J}_{b e} \boldsymbol{t}_{b}+\boldsymbol{J}_{m e} \dot{\boldsymbol{\theta}},
$$

where $\boldsymbol{t}_{b}=\left[\boldsymbol{v}_{b}^{T}, \boldsymbol{\omega}_{b}^{T}\right]^{T} \in R^{6}$ is the twist vector constituting linear velocity $\left(\boldsymbol{v}_{b}\right)$ and angular velocity $\left(\boldsymbol{\omega}_{b}\right)$ of the base, $\dot{\boldsymbol{\theta}}=\left[\begin{array}{ll}\dot{\boldsymbol{\theta}}_{1}{ }^{T} & \dot{\boldsymbol{\theta}}_{2}{ }^{T}\end{array}\right]^{T} \in R^{n}$ is the vector of manipulator joint velocities, $\boldsymbol{J}_{b e}=\left[\begin{array}{ll}\boldsymbol{J}_{b e 1}{ }^{T} & \boldsymbol{J}_{b e 2}{ }^{T}\end{array}\right]^{T} \in R^{12 \times 6}$ is the Jacobian matrix for base, and $\boldsymbol{J}_{m e}=\left[\begin{array}{cc}\boldsymbol{J}_{m e 1} & \boldsymbol{O} \\ \boldsymbol{O} & \boldsymbol{J}_{m e 2}\end{array}\right] \in R^{12 \times n}$ is the Jacobian matrix for manipulator. Note that 1 and 2 in the subscripts represent arm- 1 and arm-2, respectively, and $\boldsymbol{O}$ is the null matrix of compatible dimensions.

In (1), end-effectors' velocities $\left(\boldsymbol{t}_{e}\right)$ is represented in terms of both base velocities $\left(\boldsymbol{t}_{b}\right)$ and joint velocities $(\dot{\boldsymbol{\theta}})$. In order to obtain Jacobian which maps $\dot{\boldsymbol{\theta}}$ directly into $\boldsymbol{t}_{e}$, it is required to calculate $\boldsymbol{t}_{b}$ in terms of $\dot{\boldsymbol{\theta}}$. This is obtained from the expressions of linear momentum ( $\boldsymbol{p})$ and angular momentum $(l)$ as an extension of single-arm robot in [12], as

$$
\left[\begin{array}{c}
\boldsymbol{p} \\
\boldsymbol{l}
\end{array}\right]=\boldsymbol{I}_{b} \boldsymbol{t}_{b}+\boldsymbol{I}_{b m 1} \dot{\boldsymbol{\theta}}_{1}+\boldsymbol{I}_{b m 2} \dot{\boldsymbol{\theta}}_{2}+\left[\begin{array}{c}
\mathbf{0} \\
\boldsymbol{c}_{0} \times \boldsymbol{p}
\end{array}\right] .
$$

In (2), $\boldsymbol{I}_{b} \in R^{6 \times 6}$ is the inertia matrix of the floatingbase, and $\boldsymbol{I}_{b m 1}, \boldsymbol{I}_{b m 2} \in R^{6 \times(n / 2)}$ are the coupling inertia matrices for arm-1 and arm-2, respectively. Substituting $\boldsymbol{t}_{b}$ from (2) into (1), one obtains

$\boldsymbol{t}_{e}=\left(\boldsymbol{J}_{m e}-\boldsymbol{J}_{b e} \boldsymbol{I}_{b}^{-1} \boldsymbol{I}_{b m}\right)\left[\begin{array}{c}\dot{\boldsymbol{\theta}}_{1} \\ \dot{\boldsymbol{\theta}}_{2}\end{array}\right]+\boldsymbol{J}_{b e} \boldsymbol{I}_{b}^{-1}\left[\begin{array}{c}\boldsymbol{p} \\ \boldsymbol{l}-\boldsymbol{c}_{0} \times \boldsymbol{p}\end{array}\right]$,

where $\boldsymbol{I}_{b m}=\left[\begin{array}{ll}\boldsymbol{I}_{b m 1} & \boldsymbol{I}_{b m 2}\end{array}\right]$. If no external force is acting on the base, and the system starts from rest, $\boldsymbol{p}=\boldsymbol{l}=\mathbf{0}$, and

$$
\boldsymbol{t}_{e}=\boldsymbol{J}_{g} \dot{\boldsymbol{\theta}} \text {, where } \boldsymbol{J}_{g}=\left(\boldsymbol{J}_{m e}-\boldsymbol{J}_{b e} \boldsymbol{I}_{b}^{-1} \boldsymbol{I}_{b m}\right) \text {. }
$$

In (4), $\boldsymbol{J}_{g}$ is referred to as Generalized Jacobian Matrix (GJM) [10]. The GJM in (4) is different than the Jacobian of the earth-based manipulator as the former contains inertia terms. It will be used for reactionless visual servoing of the dual-arm space robot in the subsequent sections.

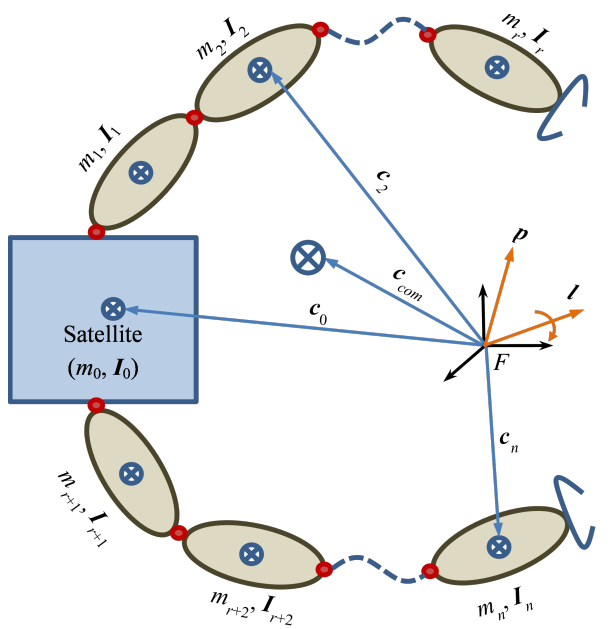

Fig. 1. A dual-arm robot mounted on a service satellite

\section{Visual Servoing of a Dual-arm Space Robot}

In contrast to the earth-based dual-arm robot, the dualarm space robot has coupled motion of arms and base satellite. This is evident from (4) where mapping between end-effectors' velocity and joint rates is no more function of mere kinematic parameters. Therefore, this section emphasizes on visual servoing of a dual-arm space robot without reactionless manipulation.

Visual servoing uses signals extracted from visual information as a feedback to close the control loop [17]. The dual-arm robot carries one camera each on both arms, hence, the visual servoing control law can be defined as

$$
\boldsymbol{t}_{c}=-\lambda \boldsymbol{L}_{\mathrm{s}}^{+} \boldsymbol{e}
$$

where $\boldsymbol{L}_{S} \in R^{2 N \times 12}$ is the image Jacobian or interaction matrix, $\boldsymbol{t}_{c}$ is the camera velocity, $\lambda$ is a scalar gain which determines the speed of convergence of the visual servoing, and $e$ is the error between the current features $(s)$ and the desired features $\left(s^{*}\right) . \boldsymbol{L}_{S}$ and $\boldsymbol{t}_{c}$ have the following representation:

$$
\boldsymbol{t}_{c}=\left[\begin{array}{c}
\boldsymbol{t}_{c 1} \\
\boldsymbol{t}_{c 2}
\end{array}\right], \text { and } \boldsymbol{L}_{S}=\left[\begin{array}{cc}
\boldsymbol{L}_{s 1} & \boldsymbol{O} \\
\boldsymbol{O} & \boldsymbol{L}_{s 2}
\end{array}\right]
$$

In (6), $\boldsymbol{L}_{s 1}$ and $\boldsymbol{L}_{s 2}$ are the image Jacobians for arm-1 and arm-2, respectively, and $\boldsymbol{t}_{c i}=\left[\boldsymbol{v}_{c i}^{T}, \boldsymbol{\omega}_{c i}^{T}\right]^{T}$ is the twist vector of the camera- $i$, for $i=1,2$. It is convenient to obtain feature velocities in terms of joint velocities as the actuators are placed at the joints.

For the dual-arm space robot, it is not possible to decouple motion of the arms, and obtain two independent Jacobians. As discussed in Section 2, the joint velocities of the space robot can be mapped into end-effector velocities using the GJM, $\boldsymbol{J}_{\boldsymbol{g}}$, as given in (4). Therefore, (5) can be rewritten in terms of joint velocities of arm-1 and arm-2 using (4) as

$$
\left[\begin{array}{c}
\dot{\boldsymbol{\theta}}_{1} \\
\dot{\boldsymbol{\theta}}_{2}
\end{array}\right]=-\lambda \boldsymbol{J}_{1}^{+}\left[\begin{array}{c}
\dot{\boldsymbol{e}}_{1} \\
\dot{\boldsymbol{e}}_{2}
\end{array}\right]
$$


where the modified image Jacobian $\boldsymbol{J}_{1}=\boldsymbol{L}_{S} \boldsymbol{J}_{g}$. It is worth noting that even though $\dot{\boldsymbol{\theta}}_{1}$ and $\dot{\boldsymbol{\theta}}_{2}$, obtained from the (7), will servo the manipulator to achieve the desired features, it will produce uncontrolled motion of the floating-base, i.e., base satellite. Having solution of $\dot{\boldsymbol{\theta}}=\left[\dot{\boldsymbol{\theta}}_{1}^{T} \dot{\boldsymbol{\theta}}_{1}^{T}\right]^{T}$, and $\boldsymbol{t}_{e}=$ $\boldsymbol{J}_{g} \dot{\boldsymbol{\theta}}$ from (4), the motion of the base-satellite is obtained using (1) as

$$
\boldsymbol{t}_{b}=\boldsymbol{J}_{b e}^{-1}\left(\boldsymbol{t}_{e}-\boldsymbol{J}_{m e} \dot{\boldsymbol{\theta}}\right) .
$$

As there is no control on the motion of the base satellite, this can result into attitude disturbance as will be shown in Section V.

\section{Reactionless Visual Servoing Control}

As discussed in the previous section, the GJM-based visual servoing in (7) leads to attitude disturbances. In this section, a solution based on task function approach is proposed to overcome the above disadvantage.

\section{A. Task Function Approach for Reactionless Visual Servoing}

Task function approach uses some DOFs for completion of visual servoing task whereas redundant DOFs are used for completion of additional tasks. In (6), $e$ is designed as a visual servoing task for the dual-arm robot. This is referred to as the primary task. On the other hand regulating the base attitude disturbance to zero is treated as the secondary task and denoted as $\boldsymbol{e}_{s}$. Using the task function approach [18], regulation of the primary and secondary robotic tasks is formulated as

$$
\boldsymbol{e}_{t}=\boldsymbol{J}_{\mathbf{1}}^{+} \boldsymbol{e}+\beta\left(\mathcal{I}_{n}-\boldsymbol{J}_{\mathbf{1}}^{+} \boldsymbol{J}_{\mathbf{1}}\right) \boldsymbol{e}_{s},
$$

where $e_{t}$ is the total robotic task. The projection operator $\left(\mathcal{I}_{n}-\boldsymbol{J}_{\mathbf{1}}^{+} \boldsymbol{J}_{\mathbf{1}}\right)$ ensures that the realization of the secondary task, $\boldsymbol{e}_{s}$, does not affect the main task, $\boldsymbol{e}$. In fact, the columns of the operator $\left(\mathcal{I}_{n}-\boldsymbol{J}_{1}^{+} \boldsymbol{J}_{1}\right)$ belongs to the null space of $\boldsymbol{J}_{1}$. In (9), the value of $\beta$ is critical and needs to be accurately tuned [15]. Large value of $\beta$ will result into some oscillations, while too small values may cause a steady state error in the end effector velocity. The proposed solution to the reactionless visual servoing takes care of tuning of $\beta$. It is done automatically within the optimization framework that defines the secondary task. Finally, the visual servoing control law in terms of joint velocities is obtained as

$$
\dot{\boldsymbol{\theta}}=-\lambda \boldsymbol{e}_{t} .
$$

\section{B. Defining the Secondary Task}

The secondary task is traditionally used in the robotic literature to satisfy a set of constraints in addition to the main task to be completed. These constraints are represented using a cost function whose value is null when these constraints are satisfied. Hence, the gradient of the cost function is used as a secondary task to be regulated to zero during the main task, i.e., the servoing task [15]. For the satellite mounted dual-arm robot under study, the objective is to perform the servoing without any base reaction moment. Hence, it will be referred to as reactionless visual servoing. It is assumed that the satellite is free to move along Cartesian axes. In order to obtain constraints for reactionless manipulation, (2) is first rewritten only in terms of $\boldsymbol{\omega}_{0}$. Note that matrices $\boldsymbol{I}_{b}$ and $\boldsymbol{I}_{b m}$ in (2) have the following block representation:

$$
\boldsymbol{I}_{b}=\left[\begin{array}{cc}
\boldsymbol{I}_{b, v} & \boldsymbol{I}_{b, c}^{T} \\
\boldsymbol{I}_{b, c} & \boldsymbol{I}_{b, \omega}
\end{array}\right] ; \boldsymbol{I}_{b m}=\left[\begin{array}{ll}
\boldsymbol{I}_{b m 1, v} & \boldsymbol{I}_{b m 2, v} \\
\boldsymbol{I}_{b m 1, \omega} & \boldsymbol{I}_{b m 2, \omega}
\end{array}\right] \text {. }
$$

Using (2) and (11), the expression of angular momentum $\boldsymbol{l}$ in (2) can also be reformulated in terms of $\boldsymbol{\omega}_{0}$ as

$$
\boldsymbol{l}=\tilde{\boldsymbol{I}}_{b} \boldsymbol{\omega}_{0}+\tilde{\boldsymbol{I}}_{b m 1} \dot{\boldsymbol{\theta}}_{1}+\tilde{\boldsymbol{I}}_{b m 2} \dot{\boldsymbol{\theta}}_{2}+\boldsymbol{c}_{c o m} \times \boldsymbol{p},
$$

where

$$
\tilde{\boldsymbol{I}}_{b}=\boldsymbol{I}_{b, \omega}-\boldsymbol{I}_{b, v}^{-1} \boldsymbol{I}_{b, c} \boldsymbol{I}_{b, c}^{T} ; \tilde{\boldsymbol{I}}_{b m i}=\boldsymbol{I}_{b m i, \omega}-\boldsymbol{I}_{b, v}^{-1} \boldsymbol{I}_{b, c} \boldsymbol{I}_{b m i, v} .
$$

As the angular momentum is conserved and system starts from the rest, i.e., $\boldsymbol{l}=\boldsymbol{p}=\mathbf{0}$, (12) simplifies to

$$
\tilde{\boldsymbol{I}}_{b} \boldsymbol{\omega}_{0}+\tilde{\boldsymbol{I}}_{b m 1} \dot{\boldsymbol{\theta}}_{1}+\tilde{\boldsymbol{I}}_{b m 2} \dot{\boldsymbol{\theta}}_{2}=\mathbf{0} .
$$

If stationary state of the attitude of the base is maintained, i.e., $\boldsymbol{\omega}_{0}=0$, then

$$
\tilde{\boldsymbol{I}}_{b m 1} \dot{\boldsymbol{\theta}}_{1}+\tilde{\boldsymbol{I}}_{b m 2} \dot{\boldsymbol{\theta}}_{2}=\mathbf{0} .
$$

where $\tilde{\boldsymbol{I}}_{b m i}$ is referred to as coupling angular momentum. It is clear from (15) that for a dual-arm robot, it is sum of the coupling angular momenta of both arms, not of the individual arms, has to be zero for reactionless manipulation. Hence, the secondary cost function is taken as

$$
h_{s}=\left\|\tilde{\boldsymbol{I}}_{b m} \dot{\boldsymbol{\theta}}\right\|,
$$

where $\tilde{\boldsymbol{I}}_{b m}=\left[\begin{array}{ll}\tilde{\boldsymbol{I}}_{b m 1} & \tilde{\boldsymbol{I}}_{b m 2}\end{array}\right]$ and $\dot{\boldsymbol{\theta}}=\left[\begin{array}{ll}\dot{\boldsymbol{\theta}}_{1}^{T} & \dot{\boldsymbol{\theta}}_{2}^{T}\end{array}\right]^{T}$.

Classical way to define the secondary task is the gradient of the cost function, i.e. $\boldsymbol{e}_{s}=\partial h s / \partial \boldsymbol{\theta}$. However, here, $h_{s}$ is nonholonomic and function of $\dot{\boldsymbol{\theta}}$, which is also the output of visual servoing controller (10). Hence, the gradient cannot be derived in terms of $\boldsymbol{\theta}$.

There are many alternatives to avoid the direct analytical computation of the gradient [19]. For example, the gradient can be locally estimated using numerical methods. Another method is to consider the secondary task as a design variable $\tilde{e}_{s}$. This variable is estimated in such a way that it minimizes the cost function $h_{s}$. i.e.,

$$
\tilde{\boldsymbol{e}}_{\boldsymbol{s}}=\arg \min _{\boldsymbol{e}_{s}}\left(h_{s}\right) .
$$

Using (9), (16) and (10), (17) can be rewritten as follows:

$$
\tilde{\boldsymbol{e}}_{\boldsymbol{s}}=\arg \min _{\boldsymbol{e}_{s}}\left(\left\|-\lambda \tilde{\boldsymbol{I}}_{b m}\left(\boldsymbol{J}_{\mathbf{1}}^{+} \boldsymbol{e}+\beta\left(\mathcal{I}_{n}-\boldsymbol{J}_{\mathbf{1}}^{+} \boldsymbol{J}_{\mathbf{1}}\right) \boldsymbol{e}_{\boldsymbol{s}}\right)\right\|\right)
$$

where $\boldsymbol{\beta} \boldsymbol{e}_{s}=\tilde{e}_{s}$, and $\beta$ is set to one. In other words, the variable $\beta$ is augmented with the secondary task $\boldsymbol{e}_{s}$ as design parameter. The local optimization estimates both of them as the parameter $\tilde{e}_{s}$ that minimize the cost function $h_{s}$. As mentioned earlier, the parameter $\beta$ is critical since it affects the behaviour of the visual servoing control law. In this work, no special procedure is required to estimated value of $\beta$.

Efficacy of the proposed approach for reactionless visual serving of a 6-DOF planar dual-arm robot is illustrated in the next section. 


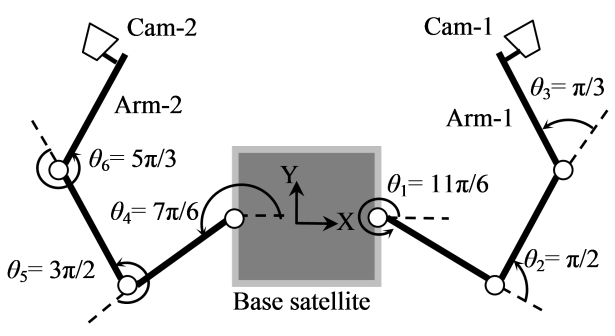

Fig. 2. Schematic of a 6-DOF planar dual-arm space robot

TABLE I

MODEL PARAMETERS OF THE SATELLITE AND DUAL-ARM ROBOT

\begin{tabular}{ccccc}
\hline \hline & Satellite & \multicolumn{3}{c}{ Arm-1 and 2 } \\
& & Link-1 & Link-2 & Link-3 \\
\hline mass $(\mathrm{Kg})$ & 500 & 10 & 10 & 10 \\
length $(\mathrm{m})$ & $1 \times 1$ & 1 & 1 & 1 \\
$I_{z z}\left(\mathrm{Kg} \cdot \mathrm{m}^{2}\right)$ & 83.61 & 1.05 & 1.05 & 1.05 \\
\hline
\end{tabular}

\section{Results AND Discussions}

In order to illustrate the proposed reactionless visual servoing, a 6-DOF planar dual-arm robotic system mounted on a service satellite is considered, as shown in Fig. 2 . The model parameters of the dual-arm and satellite are shown in Table I. Each manipulator has 3-DOF and three links connected by the revolute joints. The dual-arm is placed in XY-plane, and the relative joint angles for both arms are shown in Fig. 2. The end-effector of each arm is mounted with a camera. Two points to be tracked are placed at $(-0.5 m, 2 m,-0.1 m)$ and $(-0.5 m, 2 m, 0.1 m)$. The cameras observe the point features and the image coordinates are extracted for the purpose of visual servoing. The desired features are calculated based on the desired pose of camera- 1 and camera- 2 as $(-0.14 m, 1.78 m, 2.56 \mathrm{rad})$ and $(-0.7 \mathrm{~m}, 1.57 \mathrm{~m}, 1.04 \mathrm{rad})$, respectively. In order to validate the formulation developed in Sections III-IV, the following numerical experiments were carried out:

- Case A: Visual servoing with the GJM-based control.

- Case B: Reactionless visual servoing with the augmented GJM-based control and task function approach.

Results are obtained using space robot module of Recursive Dynamics Simulator (ReDySim) [20], and elaborated in the
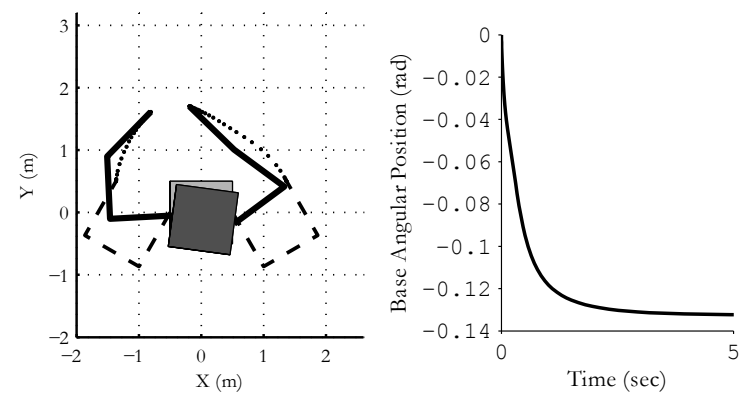

Fig. 3. Initial (dashed line) and final (solid lines) configurations of the robot (left), and angular position of the base satellite (right) in Case A
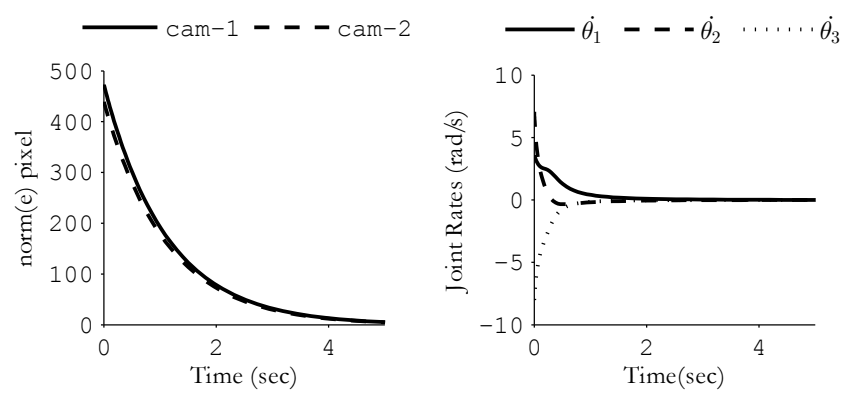

Fig. 4. Norm of pixel error and joint rates of arm-1 in Case A
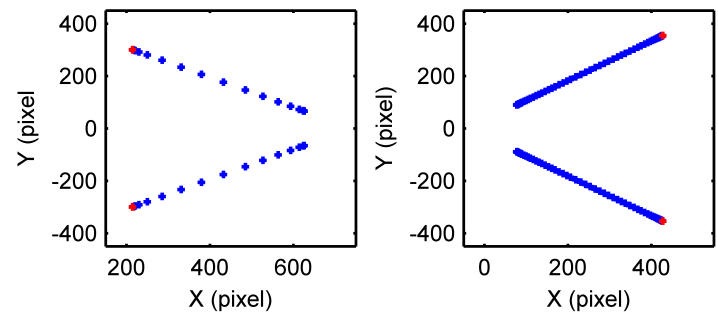

Fig. 5. Variation of features for arm-1 (left) and arm-2 (right) in Case A

next subsections.

\section{A. Case A: Visual servoing with the GJM-based control}

In order to validate theory developed in Section III, and to highlight the problems that needs to be mitigated in the reactionless visual servoing, the GJM-based visual servoing of the dual-arm robot is presented in this section. Here, the attitude controller is assumed to be shut-off, and the base satellite works in the free-floating mode. Next, the GJMbased control law derived in (7) is applied. Since there is no external force acting on the satellite, the total linear and angular momenta are conserved. Therefore, any reaction due to motion of the dual-arm causes change in the orientation of the base satellite which is evident from Fig. 3, where the maximum change in the orientation is $0.13 \mathrm{rad}$, which is not desired. Figure 4, however, shows successful visual servoing where both norm of pixel error and joint rates for arm-1 converge to zero. Moreover, observed features (in blue) move towards the desired features (in red) as depicted in Fig. 5.
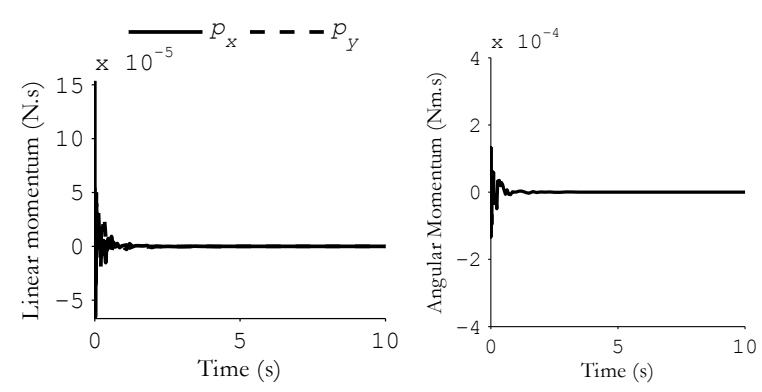

Fig. 6. Conservation of linear and angular momenta in Case A. 
In order to validate the results of numerical experiment, the linear and angular momenta are plotted in Fig. 6. It can be seen that they are of order $10^{-4}$. This confirms correctness of the simulation results as momentum is conserved.

\section{B. Case B: Reactionless Visual Servoing}

The problem of base attitude disturbance with the GJMbased visual servoing has been brought forward through the experiment illustrated in the previous section. In order to overcome this disadvantage, the task function approach proposed in Section IV is implemented for reactionless visual servoing. For this, optimal control law derived in (18), which ensures that the motion of the dual-arm does not affect the base attitude, is used. It is worth noting that the modified image Jacobian $\boldsymbol{J}_{1} \in R^{8 \times 6}$ has maximum rank equals to 6 . Here, as the two feature points are along vertical line in the image plane, and the robot moves in a plane perpendicular to the vertical line, the rank of $\boldsymbol{J}_{1}$ is reduced by two, i.e., $\operatorname{rank}\left(\boldsymbol{J}_{1}\right)=4$. Hence, there exits a null space, dimension of which is 2 . This is utilized in designing the secondary task. Figure 7 shows that the change in the orientation of the base satellite is $1.22 \times 10^{-6} \mathrm{rad}$ using the proposed method. This shows significant improvement in comparison to the previous subsection where the change in attitude of the base satellite was $0.13 \mathrm{rad}$. It can also be seen from Fig. 8 that the norm of pixel error showing the performance of primary task, and joint rates reduce to zero proving successful visual servoing. This is also depicted in Fig. 9 where the observed feature (in blue) approached the desired feature (in red). Cost of secondary task, i.e., coupling angular momentum $\left(\tilde{\boldsymbol{I}}_{b m} \dot{\boldsymbol{\theta}}\right)$, is also plotted in Fig. 10 with dashed line and compared with the same for Case A, discussed in the previous subsection. It can be seen that the maximum value of $\tilde{\boldsymbol{I}}_{b m} \dot{\boldsymbol{\theta}}$ was $160.55 \mathrm{Nm} . \mathrm{s}$ in the case of mere GJM-based control (Case A) on the other hand it was reduced to only $1.7 \times 10^{-4} \mathrm{Nm}$.s in the case of reactionless visual servoing (Case B). This shows efficacy of the proposed control.

\section{Performance Evaluation of the Proposed Algorithm}

In order to evaluate performance of the proposed reactionless visual servoing (Case B) in minimizing the base attitude disturbance, six more numerical experiments have been carried out with different initial configurations and
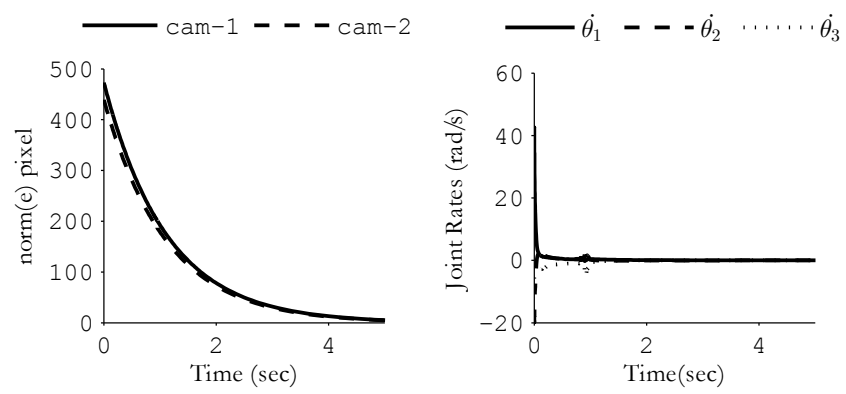

Fig. 8. Norm of pixel error and joint rates of arm-1 in Case B
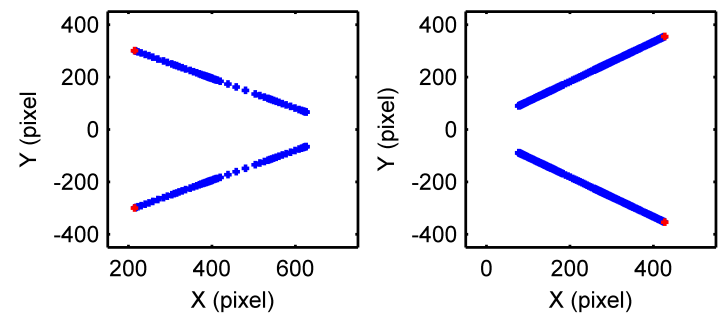

Fig. 9. Variation of features for arm-1 (left) and arm-2 (right) in Case B.

location of the feature points. The results are compared with the GJM-based visual servoing (Case A) for the norm of pixel error, maximum coupling angular momentum and maximum change in the base orientation as shown in Table II. Note that the first experiment is the one discussed in the previous two subsections.

Table II clearly shows that for Case B, the end result of primary task, i.e., pixel error, is not influenced by both presence of the secondary task and variation of initial conditions. This shows robustness of the method in completing the primary task. It can also be seen that in Case A the maximum value of coupling angular momentum was $1414.6 \mathrm{Nm} . \mathrm{s}$ (Experiment 5), where as the same for case B was 251.52 $1.7 \times 10^{-4}$ Nm.s. This shows effectiveness of the proposed approach in successful completion of the secondary task in all the seven experiments. This fact is also evident from the values of maximum change in the attitude of the base satellite, which was limited to $0.5 \mathrm{rad}$ in Case A, whereas the
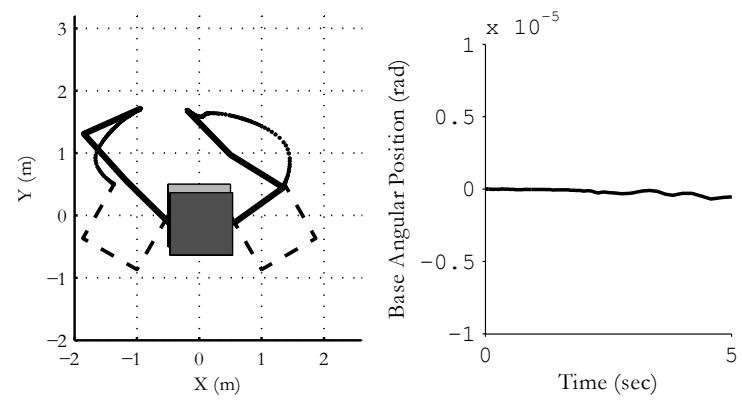

Fig. 7. Initial (dashed line) and final (solid lines) configurations of the robot (left), and angular position of the base satellite (right) in Case B.

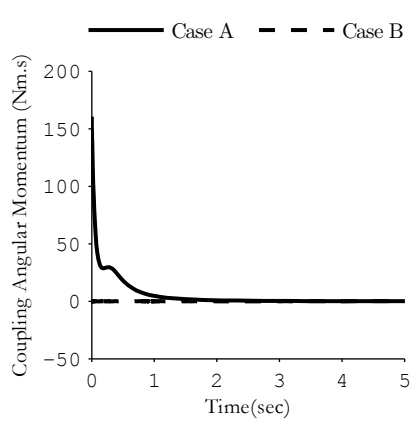

Fig. 10. Variation of secondary cost function $h_{s}=\tilde{\boldsymbol{I}}_{b m} \dot{\boldsymbol{\theta}}$ 
TABLE II

PERFormance EVALUATION OF THE PROPOSED ALGORITHM ( $\mathrm{T}=10 \mathrm{~s}$ )

\begin{tabular}{|c|c|c|c|c|c|c|c|}
\hline & & \multicolumn{2}{|c|}{$\begin{array}{c}\text { norm }(e) \\
\text { pixel }\end{array}$} & \multicolumn{2}{|c|}{$\begin{array}{c}h_{s}(\max ) \\
N m . s\end{array}$} & \multicolumn{2}{|c|}{$\begin{array}{c}\theta_{0}(\max ) \\
r a d\end{array}$} \\
\hline & & A & $\mathrm{B}$ & $\mathrm{A}$ & $\mathrm{B}$ & $\mathrm{A}$ & $\mathrm{B}$ \\
\hline 1 & Cam 1 & 0.06 & 0.06 & 160.55 & $1.7 \mathrm{E}-04$ & 0.13 & $1.2 \mathrm{E}-06$ \\
\hline \multirow{2}{*}{2} & Cam1 & 0.03 & 0.03 & & & & \\
\hline & Cam2 & 0.03 & 0.03 & 201.42 & 2.3E-04 & 0.17 & $3.6 \mathrm{E}-07$ \\
\hline \multirow{2}{*}{3} & Cam1 & 0.1 & 0.1 & 33 & $16 \mathrm{E} 04$ & 003 & 70 E 07 \\
\hline & Cam2 & 0.17 & 0.17 & 23 & $1.6 \mathrm{E}-04$ & 0.03 & $7.0 \mathrm{E}-07$ \\
\hline \multirow{2}{*}{4} & Cam 1 & 0.03 & 0.03 & 206 & $17 \mathrm{~F}_{0} 04$ & 000 & $63 F_{-07}$ \\
\hline & Cam 2 & 0.41 & 0.41 & 2.90 & $1 . / \mathrm{E}-04$ & 0.09 & $0.3 \mathrm{E}-\mathrm{U} /$ \\
\hline \multirow{2}{*}{5} & Cam1 & 0.04 & 0.04 & 14146 & 17F-04 & 050 & 7 2 \\
\hline & Cam2 & 0.03 & 0.03 & 1414.6 & & & \\
\hline \multirow{2}{*}{6} & Cam 1 & 0.17 & 0.17 & 2089 & $24 \mathrm{E}-04$ & 0.03 & 13E-06 \\
\hline & Cam 2 & 0.02 & 0.02 & & & & \\
\hline \multirow{2}{*}{7} & Cam 1 & 0.08 & 0.08 & 44661 & 2.1E-04 & 0.28 & 14E-06 \\
\hline & Cam 2 & 0.1 & 0.1 & & & & \\
\hline \multirow{2}{*}{8} & Cam 1 & 0.17 & 0.17 & 22373 & 1.7E-04 & 014 & $29 \mathrm{E}-06$ \\
\hline & Cam 2 & 0 & 0 & & & & \\
\hline
\end{tabular}

maximum value of the same for Case B was $7.23 \times 10^{-7} \mathrm{rad}$. These show superiority of the proposed method.

\section{CONCLUSIONS}

In this paper a reactionless visual servoing has been proposed for a dual-arm robotic system mounted on a service satellite. Alignment of the robot's endeffectors is very important while performing any On Orbit Services (OSS). This is done through visual servoing to overcome any communication delay between the earth-based station and the satellite. The importance of the attitude control of the base satellite is emphasised in detail, and the problem is solved using the augmented Generalized Jacobian Matrix (GJM) based control and task function approach. For this, visual servoing is considered as a primary task, whereas minimization of the attitude disturbance is assumed as a secondary task. Cost function for the secondary task is formulated using conservation of angular momentum. As the cost function is nonholonomic, it is included in task function approach by formulating an optimization problem.

Effectiveness of the proposed methodology is demonstrated using a set of numerical experiments. The results are compared with the one obtained from the GJM-based visual servoing, and it was found that the proposed approach helped in reducing the attitude disturbance to zero. The correctness of the simulations is backed by plotting conservation of momentum. Finally, a set of experiments with different initial configuration and location of the features were carried out which showed performance of the algorithm in completing both the primary and secondary tasks successfully.

The proposed approach lends utility in reactionless visual servoing of under water robots and earth-based robots with flexible-base. The method can also be useful in designing a control law for mobile manipulator with lighter base for faster operation. As the proposed method uses task function approach, it yields results when there exists degree-ofredundancy to accommodate for secondary task or modified image Jacobian $\left(\boldsymbol{J}_{1}\right)$ looses its rank. Other limitation is that the method does not take into account collision and singularity avoidances which will be taken up in future. It is also planned to validate the proposed method using an earthbased prototype of a planar dual-arm robot mounted on an air-bearing table.

\section{ACKNOWLEDGEMENT}

The authors thank B. K. Dhanush, a student in NIT Trichy, for his contribution in the initial phase of the work.

\section{REFERENCES}

[1] A. Long, M. Richards, and D. E. Hastings, "On-orbit servicing: a new value proposition for satellite design and operation," Journal of Spacecraft and Rockets, vol. 44, no. 4, pp. 964-976, 2007.

[2] On-Orbit Satellite Servicing Study. National Aeronautics and Space Administration, Goddard Space Flight Center, 2010.

[3] N. Inaba and M. Oda, "Autonomous satellite capture by a space robot: world first on-orbit experiment on a japanese robot satellite ets-vii," in IEEE Int. Conf. on Robotics and Automation, vol. 2, 2000, pp. 1169-1174.

[4] A. H. Abdul Hafez and C. V. Jawahar, "Visual servoing by optimization of a $2 \mathrm{~d} / 3 \mathrm{~d}$ hybrid objective function," in IEEE Int. Conf. on Robotics and Automation, 2007, pp. 1691-1696.

[5] M. Marey and F. Chaumette, "A temptative to reach a visual singular configuration using halley's method." in IEEE Int. Conf. on Robotics and Automation, 2009, pp. 1122-1127.

[6] R. Ozawa and F. Chaumette, "Dynamic visual servoing with image moments for a quadrotor using a virtual spring approach," in IEEE Int. Conf. on Robotics and Automation, 2011, pp. 5670-5676.

[7] A. Cherubini, F. Spindler, and F. Chaumette, "A new tentacles-based technique for avoiding obstacles during visual navigation," in IEEE Int. Conf. on Robotics and Automation, 2012, pp. 4850-4855.

[8] N. Inaba, M. Oda, and M. Hayashi, "Visual servoing of space robot for autonomous satellite capture," Transactions of the Japan Society for Aeronautical and Space Sciences, vol. 46, no. 153, pp. 173-179, 2003.

[9] D. Crymble and I. Sharf, "Experiments on robotic capture of objects in space," in 55th International Astronautical Congress, 2004.

[10] S. K. Saha, "A unified approach to space robot kinematics," IEEE Transactions on Robotics and Automation, vol. 12, no. 3, pp. 401405, 1996.

[11] M. A. Torres and S. Dubowsky, "Minimizing spacecraft attitude disturbances in space manipulator systems," Journal of guidance, control, and dynamics, vol. 15, no. 4, pp. 1010-1017, 1992.

[12] K. Yoshida, K. Hashizume, and S. Abiko, "Zero reaction maneuver: flight validation with ets-vii space robot and extension to kinematically redundant arm," in IEEE Int. Conf. on Robotics and Automation, vol. 1. IEEE, 2001, pp. 441-446.

[13] S. V. Shah, I. Sharf, and A. K. Misra, "Reactionless path planning strategies for capture of tumbling objects in space using a dualarm robotic system," in AIAA Guidance, Navigation and Control Conference 2005, 2013, pp. -.

[14] E. Marchand, F. Chaumette, and A. Rizzo, "Using the task function approach to avoid robot joint limits and kinematic singularities in visual servoing," in IEEE/RSJ Int. Conf. on Intelligent Robots and Systems, vol. 3, Osaka, Japan, November 1996, pp. 1083-1090.

[15] F. Chaumette and E. Marchand, "A new redundancy-based iterative scheme for avoiding joint limits: application to visual servoing," in IEEE Int. Conf. on Robotics and Automation, vol. 2, San Francisco, USA, April 2000, pp. 1720-1725.

[16] N. Mansard and F. Chaumette, "Directional redundancy for robot control," IEEE Transactions on Automatic Control, vol. 54, no. 6, pp. 1179-1192, 2009.

[17] F. Chaumette and S. Hutchinson, "Visual servo control, part i: Basic approaches," IEEE Robotics and Automation Magazine, vol. 13, no. 4, pp. 82-90, December 2006.

[18] C. Samson, B. Espiau, and M. L. Borgne, Robot Control: The Task Function Approach. Oxford University Press, 1991.

[19] R. Fletcher, Practical methods of optimization; (2nd ed.). New York, NY, USA: Wiley-Interscience, 1987.

[20] S. V. Shah, S. K. Saha, and J. K. Dutt, Dynamics of Tree-type Robotic Systems. Springer, 2013. 